\title{
EXTENSION PROBLEM OF SUBSET-CONTROLLED QUASIMORPHISMS
}

\author{
MORIMICHI KAWASAKI \\ (Communicated by Ken Bromberg)
}

\begin{abstract}
Let $(G, H)$ be $\left(\operatorname{Ham}\left(\mathbb{R}^{2 n}\right), \operatorname{Ham}\left(\mathbb{B}^{2 n}\right)\right)$ or $\left(B_{\infty}, B_{n}\right)$. We conjecture that any semi-homogeneous subset-controlled quasimorphism on $[G, G]$ can be extended to a homogeneous subset-controlled quasimorphism on $G$ and also give a theorem supporting this conjecture by using a Bavard-type duality theorem on conjugation invariant norms.
\end{abstract}

\section{Problems and Results}

To state our conjecture, we introduce the notion of subset-controlled quasimorphism (partial quasimorphism, pre-quasimorphism) which is a generalization of quasimorphism.

Definition 1.1. Let $G$ be a group and let $H$ be a subset of $G$. We define the fragmentation norm $q_{H}$ with respect to $H$ for an element $f$ of $G$, $q_{H}(f)=\min \left\{k ; \exists g_{1} \ldots, g_{k} \in G, \exists h_{1}, \ldots h_{k} \in H\right.$ such that $\left.f=g_{1}^{-1} h_{1} g_{1} \cdots g_{k}^{-1} h_{k} g_{k}\right\}$.

If there is no such decomposition of $f$ as above, we put $q_{H}(f)=\infty$. $H$ c-generates $G$ if such decomposition as above exists for any $f \in G$.

Definition 1.2. Let $H, G^{\prime}$ be subgroups of a group $G$. A function $\mu: G^{\prime} \rightarrow \mathbb{R}$ is called an $H$-quasimorphism on $G^{\prime}$ if there exists a positive number $C$ such that for any elements $f, g$ of $G^{\prime}$,

$$
|\mu(f g)-\mu(f)-\mu(g)|<C \cdot \min \left\{q_{H}(f), q_{H}(g)\right\} .
$$

$\mu$ is called homogeneous if $\mu\left(f^{n}\right)=n \mu(f)$ holds for any element $f$ of $G^{\prime}$ and any $n \in \mathbb{Z} . \mu$ is called semi-homogeneous if $\mu\left(f^{n}\right)=n \mu(f)$ holds for any element $f$ of $G^{\prime}$ and any $n \in \mathbb{Z}_{\geq 0}$.

Such generalization as above of quasimorphism appeared first in EP06. For a symplectic manifold $(M, \omega)$, let $\operatorname{Ham}(M)$ denote the group of Hamiltonian diffeomorphisms with compact support and $\left(\mathbb{R}^{2 n}, \omega_{0}\right),\left(\mathbb{B}^{2 n}, \omega_{0}\right)$ denote the $2 n$ dimensional Euclidean space, ball with the standard symplectic form, respectively. Let $B_{n}$ denote the $n$-braid group and $B_{\infty}$ denote the infinite braid group $\bigcup_{n} B_{n}$.

We pose the following conjecture. For a group $G$, let $[G, G]$ denote the commutator subgroup (the subgroup generated by the set $\left\{[a, b]=a b a^{-1} b^{-1} \mid a, b \in G\right\}$ of commutators).

Received by the editors December 18, 2016, and, in revised form, April 19, 2017 and September 1, 2017.

2010 Mathematics Subject Classification. Primary 20J06, 53D22; Secondary 57M27.

This work was supported by IBS-R003-D1.

(C) 2018 by the author under Creative Commons Attribution-Noncommercial 3.0 License (CC BY NC 3.0) 
Conjecture 1.3. Let $(G, H)$ be $\left(\operatorname{Ham}\left(\mathbb{R}^{2 n}\right), \operatorname{Ham}\left(\mathbb{B}^{2 n}\right)\right)$ or $\left(B_{\infty}, B_{n}\right)$. For a semihomogeneous $H$-quasimorphism $\mu$ on $[G, G]$, there exists a homogeneous $H$-quasimorphism $\hat{\mu}$ on $G$ such that $\left.\hat{\mu}\right|_{[G, G]}=\mu$. In particular, any semi-homogeneous $H$-quasimorphism on $[G, G]$ is a homogeneous $H$-quasimorphism.

The author $($ Ka1 $)$ and Kimura (Ki] $)$ constructed a non-trivial $H$-quasimorphism on $G$ when $(G, H)=\left(\operatorname{Ham}\left(\mathbb{R}^{2 n}\right), \operatorname{Ham}\left(\mathbb{B}^{2 n}\right)\right),(G, H)=\left(B_{\infty}, B_{n}\right)$, respectively. Kimura also proved that the dimension of the linear space of $H$ quasimorphisms on $G$ is infinite when $(G, H)=\left(B_{\infty}, B_{n}\right)$ ([Ki2 $]$ ).

We give examples of semi-homogeneous subset-controlled quasimorphisms which are not homogeneous. Let $\mathbb{T}^{2}$ be a 2-torus. By Proposition 3.1 of [Ka3], we see that the asymptotic Oh-Schwarz invariant $\mu: \operatorname{Ham}\left(\mathbb{T}^{2}\right) \rightarrow \mathbb{R}$ with respect to the fundamental class $\left[\mathbb{T}^{2}\right]$ is a semi-homogeneous $\operatorname{Ham}(U)$-quasimorphism for any open subset $U$ of $\mathbb{T}^{2}$ whose closure $\bar{U}$ is contractible. Since a meridian curve $M$ in the 2 torus $\mathbb{T}^{2}$ is heavy but not superheavy in the sense of Entov and Polterovich ([EP09), we see that $\mu$ is not homogeneous. Let $\mathbb{A}$ be an annulus embedded to $\mathbb{T}^{2}$ such that $M, U \subset \mathbb{A}$. By restricting $\mu$ to $\operatorname{Ham}(\mathbb{A})$, we can construct a semi-homogeneous subset-controlled quasimorphism which is not homogeneous on $\operatorname{Ham}(\mathbb{A})$.

However, the author does not know whether there is a semi-homogeneous subsetcontrolled quasimorphism which is not homogeneous on $\operatorname{Ham}\left(\mathbb{B}^{2 n}\right)$.

Our main theorem is the following one which supports the above conjecture.

Theorem 1.4. Let $(G, H)$ be $\left(\operatorname{Ham}\left(\mathbb{R}^{2 n}\right), \operatorname{Ham}\left(\mathbb{B}^{2 n}\right)\right)$ or $\left(B_{\infty}, B_{n}\right)$. For a semihomogeneous $H$-quasimorphism $\mu$ on $[G, G]$ and an element $g$ of $[G, G]$ such that $\mu(g) \neq 0$, there exists a homogeneous $H$-quasimorphism $\hat{\mu}_{g}$ on $G$ such that $\hat{\mu}_{g}(g) \neq$ 0 .

In Section 2, we prepare some notions and statements. We prove Theorem 1.4 when $(G, H)=\left(B_{\infty}, B_{n}\right),\left(\operatorname{Ham}\left(\mathbb{R}^{2 n}\right), \operatorname{Ham}\left(\mathbb{B}^{2 n}\right)\right)$ in Sections 3 and 4 respectively.

\section{Preliminaries}

Let $G^{\prime}$ be a $G$-invariant subgroup of a group $G$ i.e. $g^{-1} g^{\prime} g \in G^{\prime}$ holds for any $g^{\prime} \in G^{\prime}$ and any $g \in G$. A function $\nu: G^{\prime} \rightarrow \mathbb{R}_{\geq 0}$ is called a $G$-invariant norm on $G^{\prime}$ if $\nu$ is a conjugation-invariant norm on $G^{\prime}$ (see [BIP]) and $\nu\left(g^{-1} g^{\prime} g\right)=\nu\left(g^{\prime}\right)$ holds for any $g^{\prime} \in G^{\prime}$ and any $g \in G$. A $G$-invariant norm $\nu_{0}$ on $G^{\prime}$ is called $G$-extremal if for any $G$-invariant norm $\nu$ on $G^{\prime}$, there exist $a, b \in \mathbb{R}_{\geq 0}$ such that $a \nu\left(g^{\prime}\right)-b<\nu_{0}\left(g^{\prime}\right)$ holds for any $g^{\prime} \in G^{\prime}$.

Let $G$ be a group and $H$ a subgroup of $G$ and $p, q \in \mathbb{Z}_{>0} \cup\{\infty\}$. We define the $(H, p, q)$-commutator subgroup $[G, G]_{p, q}^{H}$ of $G$ with a subgroup $H$ to be the subgroup generated by commutators $[f, g]$ such that $q_{H}(f) \leq p, q_{H}(g) \leq q$. We also define the $(H, p, q)$-commutator length $\mathrm{cl}_{p, q}^{H}:[G, G]_{p, q}^{H} \rightarrow \mathbb{R}$ by

$$
\begin{aligned}
\operatorname{cl}_{p, q}^{H}(h)=\min \{k \mid \exists & f_{1}, \cdots, f_{k}, g_{1}, \cdots, g_{k} ; \\
& \left.q_{H}\left(f_{i}\right) \leq p, q_{H}\left(g_{j}\right) \leq q(i, j=1, \cdots, k) ; h=\left[f_{1}, g_{1}\right] \cdots\left[f_{k}, g_{k}\right]\right\} .
\end{aligned}
$$

We can easily prove that $[G, G]_{p, q}^{H}$ is a $G$-invariant subgroup and $\mathrm{cl}_{p, q}^{H}$ is a $G$ invariant norm on $[G, G]_{p, q}^{H}$. To prove Theorem $[1.4$, we use the following propositions.

Proposition 2.1 ( Ka1, Ki1] $)$. Let $(G, H)$ be $\left(\operatorname{Ham}\left(\mathbb{R}^{2 n}\right), \operatorname{Ham}\left(\mathbb{B}^{2 n}\right)\right)$ or $\left(B_{\infty}, B_{n}\right)$. Then $[G, G]_{p, q}^{H}=[G, G]$ holds for any $p, q \in \mathbb{Z}_{>0} \cup\{\infty\}$. 
For a conjugation-invariant norm $\nu$ on a group $G$, let $s \nu$ denote the stabilization of $\nu$ i.e. $s \nu(g)=\lim _{n \rightarrow \infty} \frac{\nu\left(g^{n}\right)}{n}$ (this limit exists by Fekete's Lemma).

Proposition 2.2 ([Ka1 $)$. If there exists a semi-homogeneous $H$-quasimorphism $\mu$ on $[G, G]_{p, q}^{H}$ with $\mu(g) \neq 0$ for some $g \in[G, G]_{p, q}^{H}$, then $\operatorname{scl}_{p, q}^{H}(g)>0$ holds for any $p, q \in \mathbb{Z}_{>0} \cup\{\infty\}$.

Bavard ( $[\mathrm{Bav}]$ ) gave some duality theorem between stable commutator length and quasimorphisms which generalizes Matsumoto and Morita's famous work ([MM]). We use the following Bavard-type duality theorem.

Theorem 2.3 (㠃 2$)$. Let $(G, H)$ be $\left(\operatorname{Ham}\left(\mathbb{R}^{2 n}\right), \operatorname{Ham}\left(\mathbb{B}^{2 n}\right)\right)$ or $\left(B_{\infty}, B_{n}\right)$ and let $\nu$ be a conjugation-invariant norm on $G$. Then, for any element $g$ of $G$ such that $s \nu(g)>0$, there exists a homogeneous $H$-quasimorphism $\mu: G \rightarrow \mathbb{R}$ such that $\mu(g)>0$.

\section{PROOF ON BRAID GROUP}

In the present section, let $G, H$ denote $B_{\infty}, B_{n}$, respectively.

Theorem 1.4 when $(G, H)=\left(B_{\infty}, B_{n}\right)$ immediately follows from Proposition 2.2. Theorem 2.3, and the following proposition. Let $\sigma_{1}$ denote the first standard Artin generator of $B_{\infty}$. It is known that $\left\{\sigma_{1}^{ \pm 1}\right\}$ c-generates $G$.

Proposition 3.1 ([Ki1]). The restriction of $q_{\left\{\sigma_{1}^{ \pm 1}\right\}}$ to $[G, G]$ is $G$-extremal.

Proof of Theorem 1.4 when $(G, H)=\left(B_{\infty}, B_{n}\right)$. Let $g$ be an element of $[G, G]$ and let $\mu$ be a semi-homogeneous $H$-quasimorphism on $[G, G]$ with $\mu(g) \neq 0$. Since $\mu(g) \neq 0$, Proposition 2.2 implies $s c l_{p, q}^{H}(g)>0$. Thus, by Proposition 2.1 and Proposition 3.1 $s q_{\left\{\sigma_{1}^{ \pm 1}\right\}}(g)>0$. Then Theorem 2.3 implies that there exists a homogeneous $H$-quasimorphism $\hat{\mu}_{g}$ on $G$ such that $\hat{\mu}_{g}(g) \neq 0$.

\section{Proof on Hamiltonian Diffeomorphism group}

In the present section, let $G, H$ denote $\operatorname{Ham}\left(\mathbb{R}^{2 n}\right), \operatorname{Ham}\left(\mathbb{B}^{2 n}\right)$, respectively. We follow the notion of $\left[\mathrm{E}\right.$ and thus let $\phi_{F}^{t}$ denote the time- $t$ map of the Hamiltonian flow generated by $F$ for a (time-dependent) Hamiltonian function $F: \mathbb{R}^{2 n} \times[0,1] \rightarrow$ $\mathbb{R}$.

Definition 4.1 ([C], $[\mathrm{Ban}])$. The Calabi homomorphism $\mathrm{Cal}: \operatorname{Ham}\left(\mathbb{R}^{2 n}\right) \rightarrow \mathbb{R}$ is defined by

$$
\operatorname{Cal}(h)=\int_{0}^{1} \int_{M} H \omega_{0}^{n} d t \text { for a Hamiltonian diffeomorphism } h,
$$

where $H: \mathbb{R}^{2 n} \times[0,1] \rightarrow \mathbb{R}$ is a Hamiltonian function which generates $h$. Cal $(h)$ does not depend on the choice of generating Hamiltonian function $H$ and thus the functional Cal is a well-defined homomorphism.

For proving Theorem 1.4 when $(G, H)=\left(\operatorname{Ham}\left(\mathbb{R}^{2 n}\right), \operatorname{Ham}\left(\mathbb{B}^{2 n}\right)\right)$, it is important to construct a Hamiltonian analogue of $q_{\left\{\sigma_{1}^{ \pm 1}\right\}}$. Let $F: \mathbb{R}^{2 n} \rightarrow \mathbb{R}$ be a (timeindependent) Hamiltonian function such that $\phi_{F}^{1} \notin \operatorname{Ker}(\mathrm{Cal})$ and let $h$ be an element of $\operatorname{Ker}(\mathrm{Cal})$. Note that $\operatorname{Cal}\left(\phi_{F}^{t}\right)=t \operatorname{Cal}\left(\phi_{F}^{1}\right)$. We define the conjugationinvariant norm $\nu_{F, h}$ by $\nu_{F, h}=q_{\left\{\phi_{F}^{t}\right\}_{t \in \mathbb{R}} \cup\left\{h^{ \pm 1}\right\}}$. Since $[G, G]$ is a simple group and 
$[G, G]=\operatorname{Ker}(\mathrm{Cal})([\mathrm{Ban}])$, the subset $\left\{\phi_{F}^{t}\right\}_{t \in \mathbb{R}} \cup\left\{h^{ \pm 1}\right\}$ c-generates $G$. Thus $\nu_{F, h}$ is a conjugation-invariant norm on $G$.

\section{1 .}

We use the following proposition which is a Hamiltonian analogue of Proposition

Proposition 4.2. The restriction of $\nu_{F, h}$ to $[G, G]$ is $G$-extremal.

To prove Proposition 4.2, we use the following lemma.

Lemma 4.3. Let $\nu$ be a $G$-invariant norm on $[G, G]$. There exists a positive constant $C_{F, \nu}$ which depends only on $F$ and $\nu$ such that $\nu\left(\left[g, \phi_{F}^{t}\right]\right)<C_{F, \nu}$ holds for any element $g$ of $G$.

Proof. Let $R$ be a sufficient large number such that $\operatorname{Supp}(F) \subset Q_{R}$ where $Q_{R}=$ $[-R, R]^{2 n} \subset \mathbb{R}^{2 n}$. Let $h_{0}$ be an element of $[G, G]$ such that $Q_{R} \cap h_{0}\left(Q_{R}\right)=\emptyset$. Note that $\nu\left(h_{0}\right)$ depends only on $F$ and $\nu$. Fix an element $g$ of $G$ and take an element $h_{g}$ of $G$ such that $h_{g}\left(Q_{R}\right)=Q_{R}$ and $h_{g} h_{0}\left(Q_{R}\right) \cap\left(Q_{R} \cup \operatorname{Supp}(g)\right)=\emptyset$. Then $\left(h_{g} h_{0} h_{g}^{-1}\right)\left(\phi_{F}^{t}\right)^{-1}\left(h_{g} h_{0} h_{g}^{-1}\right)^{-1}$ commutes with $\phi_{F}^{t}$ and $g$ and thus $\left[g, \phi_{F}^{t}\right]=$ $\left[g,\left[\phi_{F}^{t}, h_{g} h_{0} h_{g}^{-1}\right]\right]$. Since $\nu$ is a $G$-invariant norm on $[G, G]$,

$$
\begin{aligned}
\nu\left(\left[g, \phi_{F}^{t}\right]\right) & \leq \nu\left(g\left[\phi_{F}^{t}, h_{g} h_{0} h_{g}^{-1}\right] g^{-1}\right)+\nu\left(\left[\phi_{F}^{t}, h_{g} h_{0} h_{g}^{-1}\right]^{-1}\right) \\
& =2 \nu\left(\left[\phi_{F}^{t}, h_{g} h_{0} h_{g}^{-1}\right]\right) \\
& \leq 2\left(\nu\left(\phi_{F}^{t}\left(h_{g} h_{0} h_{g}^{-1}\right)\left(\phi_{F}^{t}\right)^{-1}\right)+\nu\left(\left(h_{g} h_{0} h_{g}^{-1}\right)^{-1}\right)\right) \\
& =4 \nu\left(h_{g} h_{0} h_{g}^{-1}\right)=4 \nu\left(h_{0}\right) .
\end{aligned}
$$

Proof of Proposition 4.2. Let $\phi$ be an element of $[G, G]$ and $m$ a natural number such that $\nu_{F, h}(\phi) \leq m$. Then, by the definition of $\nu_{F, h}$, there exist $f_{1}, \ldots, f_{m} \in$ $\left\{\phi_{F}^{t}\right\}_{t \in \mathbb{R}} \cup\left\{h^{ \pm 1}\right\}$ and $g_{1}, \ldots, g_{m} \in G$ such that $\phi=g_{1}^{-1} f_{1} g_{1} \cdots g_{m}^{-1} f_{m} g_{m}$. We define a function $\tau:\left\{\phi_{F}^{t}\right\}_{t \in \mathbb{R}} \cup\left\{h^{ \pm 1}\right\} \rightarrow \mathbb{R}$ by

$$
\tau(f)= \begin{cases}t & \left(\text { if } f=\phi_{F}^{t}\right), \\ 0 & \left(\text { if } f \in\left\{h^{ \pm 1}\right\}\right) .\end{cases}
$$

We define real numbers $T_{k}(k=1, \ldots, m+1)$ by $T_{k}=\sum_{i=1}^{k-1} \tau\left(f_{i}\right)$ and set $T_{1}=0$. Then we define elements $\alpha_{k}(k=1, \ldots, m)$ of $\operatorname{Ker}(\mathrm{Cal})=[G, G]$ by

$$
\alpha_{k}= \begin{cases}{\left[\phi_{F}^{T_{k}} g_{k}^{-1}, \phi_{F}^{t_{k}}\right]} & \left(\text { if } f_{k}=\phi_{F}^{t_{k}}\right), \\ \left(\phi_{F}^{T_{k}} g_{k}^{-1}\right) f_{k}\left(\phi_{F}^{T_{k}} g_{k}^{-1}\right)^{-1} & \left(\text { if } f_{k} \in\left\{h^{ \pm 1}\right\}\right) .\end{cases}
$$

Fix a $G$-invariant norm $\nu$ on $[G, G]$. Note that Lemma 4.3 implies $\nu\left(\alpha_{k}\right) \leq$ $\max \left\{C_{F, \nu}, \nu(h)\right\}$ holds for any $k$. Since $\phi_{F}^{T_{k}} g_{k}^{-1} f_{k} g_{k}=\alpha_{k} \phi_{F}^{T_{k+1}}$ holds for any $k$,

$$
\begin{aligned}
\phi & =\phi_{F}^{T_{1}} g_{1}^{-1} f_{1} g_{1} \cdots g_{m}^{-1} f_{m} g_{m}=\alpha_{1} \phi_{F}^{T_{2}} g_{2}^{-1} f_{2} g_{2} \cdots g_{m}^{-1} f_{m} g_{m} \\
& =\alpha_{1} \alpha_{2} \phi_{F}^{T_{3}} g_{3}^{-1} f_{3} g_{3} \cdots g_{m}^{-1} f_{m} g_{m}=\ldots=\alpha_{1} \cdots \alpha_{m} \phi_{F}^{T_{m+1}},
\end{aligned}
$$

holds. Since $\phi \in \operatorname{Ker}(\mathrm{Cal})$ and $\alpha_{k} \in \operatorname{Ker}(\mathrm{Cal})$ for any $k, T_{m+1}=0$ and thus $\phi=\alpha_{1} \cdots \alpha_{m}$ holds. Since $\nu\left(\alpha_{k}\right) \leq \max \left\{C_{F, \nu}, \nu(h)\right\}$ holds for any $k, \nu(\phi) \leq$ $\max \left\{C_{F, \nu}, \nu(h)\right\} \cdot m$ holds. Hence $\nu(\phi) \leq \max \left\{C_{F, \nu}, \nu(h)\right\} \cdot \nu_{F, h}(\phi)$ holds for any element $\phi$ of $[G, G]$. 
The proof of Theorem 1.4 when $(G, H)=\left(\operatorname{Ham}\left(\mathbb{R}^{2 n}\right), \operatorname{Ham}\left(\mathbb{B}^{2 n}\right)\right)$ is completely similar to the one when $(G, H)=\left(B_{\infty}, B_{n}\right)$ if we replace Proposition 3.1 by Proposition 4.2 .

\section{ACKNOWLEDGMENT}

The author would like to thank Mitsuaki Kimura for some comments.

\section{REFERENCES}

[Ban] Augustin Banyaga, Sur la structure du groupe des difféomorphismes qui préservent une forme symplectique (French), Comment. Math. Helv. 53 (1978), no. 2, 174-227. MR 490874

[Bav] Christophe Bavard, Longueur stable des commutateurs (French), Enseign. Math. (2) 37 (1991), no. 1-2, 109-150. MR.1115747

[BIP] Dmitri Burago, Sergei Ivanov, and Leonid Polterovich, Conjugation-invariant norms on groups of geometric origin, Groups of diffeomorphisms, Adv. Stud. Pure Math., vol. 52, Math. Soc. Japan, Tokyo, 2008, pp. 221-250. MR2509711

[C] Eugenio Calabi, On the group of automorphisms of a symplectic manifold, Problems in analysis (Lectures at the Sympos. in honor of Salomon Bochner, Princeton Univ., Princeton, N.J., 1969), Princeton Univ. Press, Princeton, N.J., 1970, pp. 1-26. MR0350776

[E] M. Entov, Quasi-morphisms and quasi-states in symplectic topology, the Proceedings of the International Congress of Mathematicians (Seoul, 2014).

[EP06] Michael Entov and Leonid Polterovich, Quasi-states and symplectic intersections, Comment. Math. Helv. 81 (2006), no. 1, 75-99. MR2208798

[EP09] Michael Entov and Leonid Polterovich, Rigid subsets of symplectic manifolds, Compos. Math. 145 (2009), no. 3, 773-826. MR2507748

[Ka1] Morimichi Kawasaki, Relative quasimorphisms and stably unbounded norms on the group of symplectomorphisms of the Euclidean spaces, J. Symplectic Geom. 14 (2016), no. 1, 297-304. MR 3523258

[Ka2] Morimichi Kawasaki, Bavard's duality theorem on conjugation-invariant norms, Pacific J. Math. 288 (2017), no. 1, 157-170. MR3667767

[Ka3] M. Kawasaki, Superheavy Lagrangian immersions in surfaces, to appear in J. Symplectic Geom..

[Ki1] M. Kimura, Conjugation-invariant norms on the commutator subgroup of infinite braid group, to appear in Journal of Topology and Analysis.

[Ki2] M. Kimura, private communication.

[MM] Shigenori Matsumoto and Shigeyuki Morita, Bounded cohomology of certain groups of homeomorphisms, Proc. Amer. Math. Soc. 94 (1985), no. 3, 539-544. MR787909

Center for Geometry and Physics, Institute for Basic Science (IBS), Pohang 37673 , Republic OF KoREA

Email address: kawasaki@ibs.re.kr 